\title{
Diagnostic value of echocardiography in fetal cardiac malformation and clinical classification
}

\author{
BO WANG ${ }^{1}$, JIANNING $\mathrm{LI}^{2}$ and JUAN YIN ${ }^{1}$ \\ ${ }^{1}$ Department of Ultrasound, Jinan Maternity and Child Care Hospital; ${ }^{2}$ Department of Exceptional Lab, \\ Affiliated Hospital of Shandong University of Traditional Chinese Medicine, Jinan, Shandong 250001, P.R. China
}

Received January 21, 2019; Accepted May 16, 2019

DOI: $10.3892 /$ etm.2019.7732

\begin{abstract}
Diagnostic value of echocardiography in fetal cardiac malformation and clinical classification was investigated. In total, 206 high-risk parturients, who received a screening of prenatal fetal cardiac malformation in Jinan Maternity and Child Care Hospital from January 2015 to June 2017, were retrospectively analyzed, among those parturients, the results of labor induction or newborns of 141 parturients were diagnosed as cardiac malformation, the fetuses of 65 parturients were diagnosed as non-cardiac malformation, the detection of fetal cardiac malformation of all the parturients was carried out by two-dimensional ultrasound and four-dimensional ultrasound during gestation period, presence or absence of congenital cardiac malformation of the fetuses and clinical classification were estimated. The sensitivity of two-dimensional ultrasound diagnosis combined with four-dimensional ultrasound diagnosis was significantly higher than that of two-dimensional ultrasound diagnosis and four-dimensional ultrasound diagnosis $(\mathrm{P}<0.05)$. In addition, the sensitivity of four-dimensional ultrasound diagnosis was significantly higher than that of two-dimensional ultrasound diagnosis $(\mathrm{P}<0.05)$. The specificity and positive predictive value of four-dimensional ultrasound diagnosis were significantly higher than those of two-dimensional ultrasound diagnosis and two-dimensional ultrasound diagnosis combined with four-dimensional ultrasound diagnosis $(\mathrm{P}<0.05)$. The diagnostic coincidence rates of four-dimensional ultrasound diagnosis and two-dimensional ultrasound diagnosis combined with four-dimensional ultrasound diagnosis were significantly higher than that of two-dimensional ultrasound diagnosis $(\mathrm{P}<0.05)$. The negative predictive values of the combined ultrasound diagnosis and four-dimensional
\end{abstract}

Correspondence to: Dr Juan Yin, Department of Ultrasound, Jinan Maternity and Child Care Hospital, 2 Jingguo Xiaojingsan Road, Jinan, Shandong 250001, P.R. China

E-mail: uqf8s6@163.com

Key words: two-dimensional echocardiography, four-dimensional echocardiography, fetus, cardiac malformation, diagnostic value, clinical classification ultrasound diagnosis were significantly higher than that of two-dimensional ultrasound diagnosis $(\mathrm{P}<0.05)$. The diagnostic efficiency of two-dimensional ultrasound combined with four-dimensional ultrasound was good in the diagnosis of fetal cardiac malformation in prenatal period of pregnant women, it could improve detection rate of fetal cardiac malformation and is worthy of being generalized in clinic.

\section{Introduction}

Fetal congenital cardiac malformation is a common cause of death of perinatal infants. Fetal congenital cardiac malformation has a very negative influence on the quality of newly-born population (1). Fetal cardiac malformation is a congenital disease caused by the abnormality that appears in the formation of heart and large blood vessels of fetuses during the development of embryos (2). Cardiac malformation poses a serious threat to life and health of fetuses, most fetuses stop developing before birth, and the serious defects that appear in the development of some fetuses may have serious effects on their life even if they are born; thus, it is imperative to carry out early screening of fetal cardiac malformation (3). The most common detection method in antenatal care of pregnant women is ultrasonic testing, which has advantages such as non-invasiveness and convenient operation, in addition to clearly showing the physical condition of the fetus. Thus, ultrasonic testing plays an important role in detecting fetal cardiac malformation (4).

At present, with the development of scientific technology and medical technology, the detection rate of fetal cardiac malformation is increasing, but due to factors, such as amniotic fluid, fetal position, ventricular septal defect, etc. there are still some misdiagnosis in fetal cardiac malformation by ultrasound (5). Two-dimensional ultrasound has been widely used as a basic detection method in clinical diagnosis, however, as it is a planar image, only the image of one side of the fetus can be shown (6). However, with the development of scientific technology, four-dimensional ultrasound also has been gradually applied in clinic, and four-dimensional ultrasound can visually show the specific conditions of the heart because of its stereoscopic imaging, which helps to accurately understand the relationship between blood vessels, timely evaluate intracardiac and extracardiac hemodynamics of fetuses and easily observe fetal defects $(7,8)$. 
Although some studies have investigated the diagnostic value of ultrasound with different dimensions in fetal cardiac malformation $(9,10)$, the studies, which investigate the diagnostic value of the combined diagnosis of two-dimensional ultrasound and four-dimensional ultrasound, are rare. Therefore, the diagnostic value and clinical classification of the single detection of two-dimensional ultrasound, the single detection of four-dimensional ultrasound and their combined detection in fetal cardiac malformation were compared, in order to provide more data for the diagnosis of fetal cardiac malformation in clinic.

\section{Patients and methods}

General data. In total, 206 high-risk parturients, who received a screening of prenatal fetal cardiac malformation in the Jinan Maternity and Child Care Hospital (Jinan, China) from January 2015 to June 2017, were retrospectively analyzed, and the results of labor induction or newborns of 141 parturients were diagnosed as cardiac malformation, while the fetuses of 65 parturients were diagnosed as non-cardiac malformation. The average age of the parturients was $32.19 \pm 4.11$ years, the average gestational week was $16.21 \pm 2.42$ weeks. Inclusion criteria were: the high-risk parturients, who took prenatal examination in Jinan Maternity and Child Care Hospital and whose fetuses had cardiac malformation, were included. Exclusion criteria: the parturients, who had severe hepatorenal dysfunction, severe infections, cognitive disorder or communication disorder, and did not cooperate with the experiment. All the parturients and their family members agreed to participate in the experiment and signed the informed consent form. This study was approved by the Ethics Committee of Jinan Maternity and Child Care Hospital. The basic data are shown in Table I.

\section{Diagnostic methods}

Two-dimensional ultrasound detection. Color ultrasonic diagnostic apparatus, model number Voluson E6, produced by American GE company, was used. The probe frequency of two-dimensional ultrasound was $3.5 \mathrm{MHz}$, the probe of four-dimensional ultrasound was RAB4-8-D and the probe frequency was 2.5-7.0 $\mathrm{MHz}$. The abdomen of all the patients was exposed and the patients were in the supine position, and then placenta, amniotic fluid, spine, structure of the four limbs, abdomen, chest, neck, head and face of the fetuses were standardly sectioned and examined under two-dimensional ultrasound. Heart appearance, ventricular septum, pulmonary aorta, mitral valve and other parts of the fetuses were carefully observed, femur length, biparietal diameter, chest circumference, abdomen circumference and other parameters were recorded, the presence or absence of abnormal ultrasound was confirmed.

Four-dimensional ultrasound detection. Color ultrasonic diagnostic apparatus, model number Voluson E6, produced by GE Healthcare, was used. The probe of four-dimensional ultrasound was RAB4-8-D and the probe frequency was 2.5-7.0 MHz. All the parturients took the supine position and their abdomen was exposed, and then the four-dimensional ultrasound mode was started, the stereoscopic dynamic pictures of the fetuses could be seen. The probe was placed
Table I. General data.

\begin{tabular}{lc}
\hline & $\begin{array}{c}\text { Parturients } \\
\mathrm{n}=206\end{array}$ \\
Items & \\
\hline Age (year) & 122 \\
$\geq 30$ & 84 \\
$<30$ & \\
BMI (kg/m $\left.{ }^{2}\right)$ & 109 \\
$\geq 21$ & 97 \\
$<21$ & \\
Educational level & 165 \\
$\geq$ Middle school & 41 \\
$<$ Middle school & \\
Coagulation function & $28.31 \pm 2.75$ \\
APTT (sec) & $11.82 \pm 1.05$ \\
PT (sec) & $3.29 \pm 0.23$ \\
FIB (g/l) & $14.11 \pm 1.33$ \\
TT (sec) & $16.21 \pm 2.42$ \\
Average gestational week & \\
Liver function indicators & \\
Serum total protein $(\mathrm{g} / \mathrm{l})$ & $70.21 \pm 2.42$ \\
Glutamic-pyruvic transaminase $(\mu \mathrm{mol} / \mathrm{l})$ & $28.21 \pm 4.61$ \\
Total bilirubin $(\mu$ mol/l) & $11.24 \pm 2.12$ \\
Renal function indicators & \\
Creatinine $(\mu$ mol/l) & \\
Serum urea $(\mu \mathrm{mol} / \mathrm{l})$ & \\
Uric acid $(\mu$ mol/l) & \\
& \\
& \\
& \\
& \\
&
\end{tabular}

in the area that needed to be probed, then $\mathrm{x}$-axes, $\mathrm{y}$-axes and $\mathrm{z}$-axes were adjusted to appropriate position, the stereoscopic dynamic pictures of the fetuses were collected and carefully observed, and the function-rotation, was used to detect the fetuses when they were found to have an improper position, the detection focused on the heart and lung of the fetuses, the areas that seemed to have lesion were specifically filmed and recorded, and then the presence or absence of cardiac malformation of the fetuses was evaluated according to the images.

Observation indicators. According to the diagnostic results of labor induction or newborns, the sensitivity, specificity, diagnostic coincidence rate, positive predictive value and negative predictive value of the single diagnosis of two-dimensional ultrasound, the single diagnosis of four-dimensional ultrasound and their combined diagnosis in fetal cardiac malformation, as well as the diagnosis results of different clinical classifications, were compared.

Statistical analysis. In this experiment, SPSS 19.0 (Shanghai Cabit Information Technology Co., Ltd., Shanghai, China) statistical software was used to analyze the experimental data. Chi-square test was used in the enumeration data, $t$-test was used in the measurement data, $\mathrm{P}<0.05$ was considered to be statistically significant. 
Table II. Comparison among the results of prenatal ultrasound examination, labor induction and pregnancy [n (\%)].

\begin{tabular}{lcccccc}
\hline & $\begin{array}{c}\text { The results of } \\
\text { labor induction } \\
\text { or pregnancy }\end{array}$ & $\begin{array}{c}\text { Two-dimensional } \\
\text { ultrasound } \\
\mathrm{n}=100\end{array}$ & $\begin{array}{c}\text { Four-dimensional } \\
\text { ultrasound } \\
\mathrm{n}=120\end{array}$ & $\begin{array}{c}\text { The combined } \\
\text { ultrasound } \\
\mathrm{n}=135\end{array}$ & $\chi^{2}$ test & P-value \\
\hline $\begin{array}{l}\text { Classification } \\
\text { Downward displacement of }\end{array}$ & 13 & $8(8.00)$ & $10(8.33)$ & $13(9.63)$ & 0.228 & 0.892 \\
$\begin{array}{l}\text { Transposition of aorta } \\
\text { Atrioventricular septal defect }\end{array}$ & 10 & $7(7.00)$ & $8(6.67)$ & $9(6.67)$ & 0.013 & 0.994 \\
Pulmonary valve stenosis & 15 & $10(10.00)$ & $12(10.00)$ & $14(10.37)$ & 0.013 & 0.994 \\
Ventricular septal defect & 28 & $10(10.00)$ & $10(8.33)$ & $12(8.89)$ & 0.189 & 0.910 \\
Tetralogy of Fallot & 24 & $22(22.00)$ & $25(20.83)$ & $27(20.00)$ & 0.140 & 0.933 \\
Single ventricle & 9 & $5(20.00)$ & $23(19.17)$ & $23(17.04)$ & 0.373 & 0.830 \\
Single atrium & 11 & $6(6.00)$ & $7(5.83)$ & $9(6.67)$ & 0.289 & 0.866 \\
Other malformation & 19 & $12(12.00)$ & $17(14.17)$ & $18(13.33)$ & 0.183 & 0.913 \\
\hline
\end{tabular}

Table III. Analysis of the efficiency of two-dimensional ultrasound diagnosis.

\begin{tabular}{lrcc}
\hline Diagnostic results & Pathogenesis (malformation) & Pathogenesis (not malformation) & Summation \\
\hline Diagnosis (malformation) & 100 & 14 & 114 \\
Diagnosis (not malformation) & 41 & 51 & 92 \\
Summation & 141 & 65 & 206 \\
\hline
\end{tabular}

\section{Results}

Diagnostic results of fetal cardiac malformation in two-dimensional ultrasound, four-dimensional ultrasound and the combined ultrasound before prenatal period and the results of labor induction or pregnancy. The ratio between the number of cardiac malformation and clinical classification detected by two-dimensional ultrasound, four-dimensional ultrasound and two-dimensional ultrasound combined with four-dimensional ultrasound and the total number of cardiac malformation detected by this method was not statistically significant $(\mathrm{P}>0.05)$ (Table II).

Analysis of the efficiency of two-dimensional ultrasound diagnosis, four-dimensional ultrasound diagnosis, and the combined ultrasound diagnosis. Compared with the fetuses who were born by labor induction and the fetuses who were diagnosed with cardiac malformation after birth, there were 100 cases which were diagnosed with malformation by two-dimensional ultrasound, 120 cases which were diagnosed with malformation by four-dimensional ultrasound, and 135 cases which were diagnosed with malformation by two-dimensional ultrasound combined with four-dimensional ultrasound. The sensitivity, specificity, diagnostic coincidence rate, negative predictive value, and positive predictive value of two-dimensional ultrasound diagnosis were 70.92, $78.46,73.30,55.43$ and $87.72 \%$, respectively; the sensitivity, specificity, diagnostic coincidence rate, negative predictive value, and positive predictive value of four-dimensional ultrasound diagnosis were 85.11, 89.23, 86.41, 73.42 and $94.49 \%$, respectively; the sensitivity, specificity, diagnostic coincidence rate, negative predictive value and positive predictive value of two-dimensional ultrasound diagnosis combined with four-dimensional ultrasound diagnosis were $95.74,67.69,86.89,88.00$ and $86.54 \%$, respectively. The sensitivity of two-dimensional ultrasound diagnosis combined with four-dimensional ultrasound diagnosis was significantly higher than that of two-dimensional ultrasound diagnosis and four-dimensional ultrasound diagnosis, the difference was statistically significant $(\mathrm{P}<0.05)$. The sensitivity of four-dimensional ultrasound diagnosis was significantly higher than that of two-dimensional ultrasound diagnosis, the difference was statistically significant $(\mathrm{P}<0.05)$. The specificity and positive predictive value of four-dimensional ultrasound diagnosis were significantly higher than those of two-dimensional ultrasound diagnosis and two-dimensional ultrasound diagnosis combined with four-dimensional ultrasound diagnosis, the difference was statistically significant $(\mathrm{P}<0.05)$. There was no significant difference between the diagnostic coincidence rate of four-dimensional ultrasound diagnosis and two-dimensional ultrasound diagnosis combined with four-dimensional ultrasound diagnosis $(\mathrm{P}>0.05)$, but the diagnostic coincidence rates of four-dimensional ultrasound diagnosis and two-dimensional ultrasound diagnosis combined with four-dimensional ultrasound diagnosis were significantly higher than that of two-dimensional ultrasound diagnosis, the difference was statistically significant $(\mathrm{P}<0.05)$. The negative predictive values of the combined ultrasound diagnosis and four-dimensional ultrasound diagnosis were significantly higher than that of two-dimensional ultrasound diagnosis, the difference was statistically significant $(\mathrm{P}<0.05)$ (Tables III-VI). 
Table IV. Analysis of the efficiency of four-dimensional ultrasound diagnosis.

\begin{tabular}{lccr}
\hline Diagnostic results & Pathogenesis (malformation) & Pathogenesis (not malformation) & Summation \\
\hline Diagnosis (malformation) & 120 & 7 & 127 \\
Diagnosis (not malformation) & 21 & 58 & 79 \\
Summation & 141 & 65 & 206 \\
\hline
\end{tabular}

Table V. Analysis of the efficiency of the combined ultrasound diagnosis.

\begin{tabular}{lccr}
\hline Delivery results & Diagnosis (malformation) & Diagnosis (not malformation) & Summation \\
\hline Malformation & 135 & 6 & 141 \\
Not malformation & 21 & 44 & 65 \\
Summation & 156 & 50 & 206 \\
\hline Diagnostic results & Pathogenesis (malformation) & Pathogenesis (not malformation) & Summation \\
\hline Diagnosis (malformation) & 135 & 21 & 156 \\
Diagnosis (not malformation) & 6 & 44 & 50 \\
Summation & 141 & 65 & 206 \\
\hline
\end{tabular}

Table VI. Analysis of the efficiency of two-dimensional ultrasound, four-dimensional ultrasound and two-dimensional ultrasound combined with four-dimensional ultrasound (\%).

\begin{tabular}{lcccrr}
\hline Diagnostic value & $\begin{array}{c}\text { Two-dimensional } \\
\text { ultrasound }\end{array}$ & $\begin{array}{c}\text { Four-dimensional } \\
\text { ultrasound }\end{array}$ & $\begin{array}{c}\text { The combined } \\
\text { ultrasound }\end{array}$ & $\chi^{2}$ test & P-value \\
\hline Sensitivity & 70.92 & 85.11 & 95.74 & 32.42 & $<0.001$ \\
Specificity & 78.46 & 89.23 & 67.69 & 8.922 & $<0.050$ \\
Diagnostic coincidence rate & 73.30 & 86.41 & 86.89 & 15.90 & $<0.001$ \\
Negative predictive value & 55.43 & 73.42 & 88.00 & 17.14 & $<0.001$ \\
Positive predictive value & 87.72 & 94.49 & 86.54 & 5.166 & 0.076 \\
\hline
\end{tabular}

\section{Discussion}

Congenital cardiac malformation is a common disease caused by congenital factors (11). There are many reasons for cardiac malformation, such as chromosomal abnormality and heredity, and with the advancement of society and the development of modern industry, the exposure of pregnant women to chemicals and radiation during gestation period is higher than before, which also leads to the increase of the morbidity of fetal cardiac malformation (12-14). In general, the condition of cardiac malformation of fetuses can only be detected by ultrasound before birth, because ultrasound is a safe, rapid and effective examination method (15). Fetuses with cardiac malformation often have some symptoms after birth, such as lung infection, slow growth, and cardiac murmur, which has a great impact on living quality of children, therefore, the detection of cardiac malformation is particularly important for fetuses (16).

Statistics on the diagnostic data of a single diagnosis of two-dimensional ultrasound and four-dimensional ultrasound and the combined diagnosis in different clinical classifications of fetal cardiac malformation, was carried out. The result showed that the number of the patients who were diagnosed by two-dimensional ultrasound in different clinical classifications of cardiac malformation was slightly lower than the number of the patients who were diagnosed by four-dimensional ultrasound, but there was no statistical difference. Also the diagnostic value of the single diagnosis of two-dimensional ultrasound and four-dimensional ultrasound and the combined diagnosis in fetal cardiac malformation was compared. The result showed that the sensitivity of two-dimensional ultrasound combined with four-dimensional ultrasound was significantly higher than that of the single detection of two-dimensional ultrasound and four-dimensional ultrasound $(\mathrm{P}<0.05)$. The sensitivity of the single detection of four-dimensional ultrasound was significantly higher than that of the single detection of two-dimensional ultrasound $(\mathrm{P}<0.05)$. The diagnostic coincidence rates of four-dimensional ultrasound and the combined detection were significantly higher than that of two-dimensional ultrasound $(\mathrm{P}<0.05)$. As a traditional ultrasonic examination method, two-dimensional ultrasound can only show a flat picture of a part of the fetus 
and can not stereoscopically image the fetus, it cannot show the heart abnormality of the fetus, which leads to limitation of two-dimensional ultrasound in diagnosis of fetal cardiac malformation $(17,18)$. As a new imaging diagnosis method, the stereoscopic imaging of four-dimensional ultrasound can show the whole structure of the heart of the fetus and can also help to observe the image from different angles, which makes it possible to carefully examine the different parts of the fetus and assess the relationship between malformed parts and the tissues and organs around them. The richness of the image of four-dimensional ultrasound is a good complement to the defects of two-dimensional ultrasound (19). Studies (20) have shown that the diagnostic value of four-dimensional ultrasound is higher than that of two-dimensional ultrasound in the diagnosis of fetal cardiac malformation, which is consistent with our findings. Furthermore, it was also found in our study that the sensitivity of the combined diagnosis of two-dimensional ultrasound and four-dimensional ultrasound was higher than that of the single detection of four-dimensional ultrasound in the diagnosis of fetal cardiac malformation, which suggested that using two-dimensional ultrasound as the basis of ultrasonic test and then performing four-dimensional ultrasound could enlarge the advantages of ultrasound in examination of fetal cardiac malformation and improve detection rate of diseases.

In conclusion, the diagnostic effect of two-dimensional ultrasound combined with four-dimensional ultrasound is good in the diagnosis of fetal cardiac malformation before prenatal period of pregnant women. Two-dimensional ultrasound combined with four-dimensional ultrasound can improve the detection rate of fetal cardiac malformation, which helps medical workers to take timely and effective measures, improves the survival rate of the fetus, and has a positive effect on implementation of good prenatal and postnatal care. It is worthwhile to generalize this method in clinic.

\section{Acknowledgements}

Not applicable.

\section{Funding}

No funding was received.

\section{Availability of data and materials}

The datasets used and/or analyzed during the present study are available from the corresponding author on reasonable request.

\section{Authors' contributions}

BW wrote the manuscript, proposed and designed the study, and provided statistical methods. JL and JY were responsible for ultrasound and observation indicators analysis. All authors read and approved the final manuscript.

\section{Ethics approval and consent to participate}

The study was approved by the Ethics Committee of Jinan Maternity and Child Care Hospital (Jinan, China). Patients who participated in this research had complete clinical data. Signed informed consents were obtained from the patients or the guardians.

\section{Patient consent for publication}

Not applicable.

\section{Competing interests}

The authors declare that they have no competing interests.

\section{References}

1. Davies RR, Pasquali SK, Jacobs ML, Jacobs JJ, Wallace AS and Pizarro C: Current spectrum of surgical procedures performed for Ebstein's malformation: An analysis of the Society of Thoracic Surgeons Congenital Heart Surgery Database. Ann Thorac Surg 96: 1703-1710, 2013.

2. Sananes N, Guigue V, Kohler M, Bouffet N, Cancellier M, Hornecker F, Hunsinger MC, Kohler A, Mager C, Neumann M, et al: Nuchal translucency and cystic hygroma colli in screening for fetal major congenital heart defects in a series of 12,910 euploid pregnancies. Ultrasound Obstet Gynecol 35: 273-279, 2010.

3. Tegnander E and Eik-Nes SH: The examiner's ultrasound experience has a significant impact on the detection rate of congenital heart defects at the second-trimester fetal examination. Ultrasound Obstet Gynecol 28: 8-14, 2006.

4. Levi S, Zhang WH, Alexander S, Viart P and Grandjean H; Eurofetus study group: Short-term outcome of isolated and associated congenital heart defects in relation to antenatal ultrasound screening. Ultrasound Obstet Gynecol 21: 532-538, 2003.

5. Eleftheriades M, Tsapakis E, Sotiriadis A, Manolakos E, Hassiakos D and Botsis D: Detection of congenital heart defects throughout pregnancy; impact of first trimester ultrasound screening for cardiac abnormalities. J Matern Fetal Neonatal Med 25: 2546-2550, 2012.

6. Zabadneh N, Santagati C, Reffo E, Biffanti R, Cerutti A, Maschietto N and Milanesi O: Usefulness of fetal three-dimensional ultrasonography for detecting of congenital heart defects and associated syndromes. Pediatr Cardiol 32: 724-736, 2011.

7. Chen CP, Shih JC, Huang MC, Liu YP, Su JW, Chern SR and Wang W: Prenatal ultrasound demonstration of scoliosis, absence of one rib, a radial club hand, congenital heart defects and absent stomach in a fetus with VACTERL association. Taiwan J Obstet Gynecol 51: 139-142, 2012.

8. Volpe P, Campobasso G, De Robertis V, Di Paolo S, Caruso G, Stanziano A, Volpe N and Gentile M: Two- and four-dimensional echocardiography with B-flow imaging and spatiotemporal image correlation in prenatal diagnosis of isolated total anomalous pulmonary venous connection. Ultrasound Obstet Gynecol 30: 830-837, 2007.

9. Messing B,Cohen SM, Valsky DV, Rosenak D, Hochner-Celnikier D, Savchev S and Yagel S: Fetal cardiac ventricle volumetry in the second half of gestation assessed by 4D ultrasound using STIC combined with inversion mode. Ultrasound Obstet Gynecol 30: 142-151, 2007.

10. Bega G, Kuhlman K, Lev-Toaff A, Kurtz A and Wapner R: Application of three-dimensional ultrasonography in the evaluation of the fetal heart. J Ultrasound Med 20: 307-316, 2001.

11. Schierz IA, Pinello G, Giuffrè M, La Placa S, Piro E and Corsello G: Congenital heart defects in newborns with apparently isolated single gastrointestinal malformation: A retrospective study. Early Hum Dev 103: 43-47, 2016.

12. Nogee JM and Jay PY: The heritable basis of congenital heart disease: Past, present, and future. Circ Cardiovasc Genet 9: 315-317, 2016.

13. Park HK and Park YH: Fetal surgery for congenital heart disease. Yonsei Med J 42: 686-694, 2001.

14. Makrydimas G, Sotiriadis A, Huggon IC, Simpson J, Sharland G, Carvalho JS, Daubeney PE and Ioannidis JP: Nuchal translucency and fetal cardiac defects: A pooled analysis of major fetal echocardiography centers. Am J Obstet Gynecol 192: 89-95, 2005. 
15. Bravo-Valenzuela NJ, Peixoto AB, Nardozza LM, Souza AS and Araujo Júnior E: Applicability and technical aspects of two-dimensional ultrasonography for assessment of fetal heart function. Med Ultrason 19: 94-101, 2017.

16. Chen KB, Gu Q, Xia T, Lu X and Zhang ZD: Three-vessel-trachea view in the diagnosis of fetal cardiac great vessel malformation. J Biol Regul Homeost Agents 32: 351-355, 2018.

17. Liu L, He Y, Li Z and Zhang L: Application of two-dimensional echocardiography combined with enhanced flow in diagnosing fetal heart malformation. Clin Exp Obstet Gynecol 41: 195-201, 2014.

18. Hu G, Zhang Y, Fan M, Wang M, Siddiqui FA, Wang Y, Sun W, Sun F, Zhang D, Lei W, et al: Evaluation of fetal cardiac valve anomalies by four-dimensional echocardiography with spatiotemporal image correlation (4DSTIC). Echocardiography 33: $1726-1734,2016$
19. Adriaanse BM, Tromp CH, Simpson JM, Van Mieghem T, Kist WJ, Kuik DJ, Oepkes D, Van Vugt JM and Haak MC: Interobserver agreement in detailed prenatal diagnosis of congenital heart disease by telemedicine using four-dimensional ultrasound with spatiotemporal image correlation. Ultrasound Obstet Gynecol 39: 203-209, 2012.

20. Zhang D, Zhang Y, Ren W, Sun F, Guo Y, Sun W, Wang Y, Huang L and Cai A: Prenatal diagnosis of fetal interrupted aortic arch type a by two-dimensional echocardiography and four-dimensional echocardiography with B-flow imaging and spatiotemporal image correlation. Echocardiography 33: 90-98, 2016.

This work is licensed under a Creative Commons Attribution-NonCommercial-NoDerivatives 4.0 International (CC BY-NC-ND 4.0) License. 